\title{
Querying by sketch geographical databases
}

\author{
Yu $\operatorname{Han}^{1, a^{*}}$ \\ ${ }^{1}$ Department of Basic Courses, Shenyang Institute of Artillery, \\ Shenyang,110162, China \\ a4947919@qq.com
}

\begin{abstract}
Keywords: Spatial Query; Topological Relations; Spatial Reasoning.
Abstract. This is where the abstract should be placed. It should consist of one paragraph giving a concise summary of the material in the article below. Replace the title, authors, and addresses with your own title, authors, and addresses. You may have as many authors and addresses as you like. It is preferable not to use footnotes in the abstract or the title; the acknowledgments of funding bodies etc. are to be placed in a separate section at the end of the text.
\end{abstract}

\section{Introduction}

Spatial data query is the basis of GIS functions. how to query a massive spatial database is currently one of the bottlenecks of spatial data management? Many spatial data query methods have been proposed such as Structural Spatial Query (SSQL), Spatial Query by Natural Language (SQNL), Visual Spatial Query (VSQ), etc.

SSQL and SQNL are query languages based on text; the description of spatial relation is obscured and easily results in the mismatch. The VSQ usage icons and interface to organize query process, but it is very difficult to accurately express spatial relation of objects only use some prepared definite icons. Therefore, the research on spatial query by sketch got value. Egenhofer[1][2] proposed the design principle of the spatial data query by sketch, discussed the representation of the sketch and the query process. In 2000, Blaser[3] discussed the interface design of spatial query by sketch, sketch processing, system design of spatial query by sketch etc.In 2005, Grifoni and Rafanelli [4][5] studied identify of sketch and the query process of spatial-query-by-sketch, and analyze the indetermination problem among them. In 2007, Caduff and Egenhofer[6] studied the application of spatial query by sketch under the wireless network, and analyze the data deliver price of the environment.

This paper proposes a spatial query method by sketch using 9-intersection model and Deep Direction-Relation Matrix. This method integrates direction relations and topological relations and can handle all data types in geographical databases.

\section{Spatial Query by Sketch}

Description. Spatial-query-by-sketch uses a touch sensitive input device-ideally a touch screen with a pen. Simulations may be obtained with mice, but sketching with these devices is more cumbersome and therefore less effective. A sketched query consists of three steps, ranging from the drawing of a spatial query to its execution against a database management system.

Step 1: The user draws with a pen or a mouse a prototype geometric configuration that matches closely the spatial situation he or she expects to retrieve from the geographic database.

Step 2: Spatial-query-by-sketch parses the sketch and distills the topology relation and directional relation among the objects from the sketch, and then stores the relations as topology relation table and directional relation table for further query.

Step 3: Spatial-query-by-sketch executes the query against the spatial database using the SBSDQ-FC algorithm this paper developed and retrieves the scenes that match the sketch.

Extraction of topological relations and directional relations. Spatial relations representation is the basis of spatial data query. According to the characteristics of spatial data and sketch querying, this paper mainly considers the topological relations and the directional relations. In order to handle 
all data types in geographical databases, we use 9-intersection model and Deep-Direction-Relation Matrix.

The 9-intersection model is one of the main model of the topological relations. The model is based on point set topology, a theory that defines the rules between two point sets $\mathrm{A}$ and $\mathrm{B}$, with $\mathrm{A}$ or $\mathrm{B}$ being either a point, line, or area. A point set of A has an interior (A0), a boundary (A), and an exterior $\left(\mathrm{A}^{-}\right)$. Topological relations between two point sets $\mathrm{A}$ and $\mathrm{B}$ are characterized by the intersection of A's interior, boundary, and exterior with the interior, boundary, and exterior of B. For two simple regions without holes, the categorization shows 8 distinct topological relations (Fig. 1).

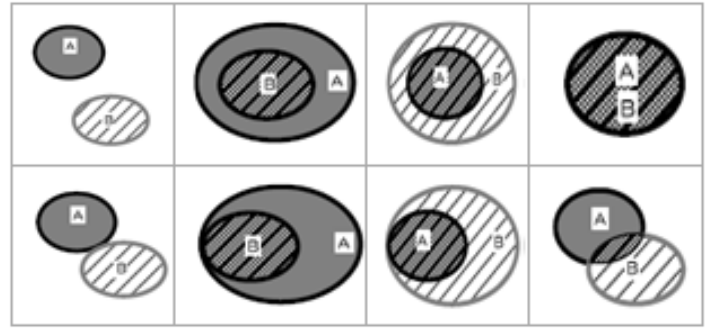

Fig. 1. the eight topological relations between two spatial regions

The Direction-Relation Matrix proposed by Goyal partitions the space into nine parts which called direction tiles. We denote those 9 direction tiles as $\{\mathrm{N}, \mathrm{S}, \mathrm{E}, \mathrm{W}, \mathrm{NE}, \mathrm{SE}, \mathrm{SW}, \mathrm{NW}, \mathrm{O}\}$, We describe direction between reference object and target object as a $3 \times 3$ matrix (Fig. 2). The value of element in the matrix either is empty (With $\emptyset$ symbolic representation), or is non-empty (With $\neg \varnothing$ symbolic representation). In 2000 Goyal extended the model to include information about the intersections of the target object with the boundaries of direction partitions which called deep direction-relation matrix. The new model is capable of recording directions between arbitrary pairs of point, line, and polygon objects. The deep direction-relation matrix is useful for direction based queries in multi-resolution spatial databases.

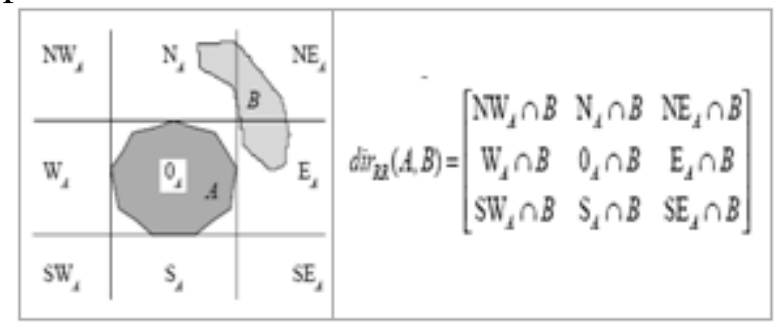

Fig. 2. the direction-relation matrix

The $\mathrm{N}$ objects in the sketch are the target objects in the query. We denote the objects as $\mathrm{V}=\{\mathrm{V} 1$, $\mathrm{V} 2, \ldots \mathrm{Vn}\}$. We can use the 9-intersection model and the deep direction-relation matrix to extract and save the topological relations and directional relations. We use array to save the relations between the objects.

We use the 9-intersection model and the deep direction-relation matrix to extract the relations between the objects in Figure 1 and establish the topological relation table and the directional relation table as Table 1 and Table 2.

Table 1. topological relations of the sketch

\begin{tabular}{llllll}
\hline & $A$ & $B 1$ & $B 2$ & $B 3$ & $C$ \\
\hline A & equal & contain & contain & disjoint & overlap \\
B1 & inside & equal & disjoint & disjoint & disjoint \\
B2 & inside & disjoint & equal & disjoint & meet \\
B3 & disjoint & disjoint & disjoint & equal & disjoint \\
C & overlap & disjoint & meet & disjoint & equal \\
\hline
\end{tabular}


Table 2. part of the directional relations of the sketch

\begin{tabular}{|c|c|c|c|c|c|c|c|c|c|c|c|c|}
\hline & \multicolumn{3}{|c|}{$A$} & \multicolumn{3}{|c|}{$B 1$} & \multicolumn{3}{|c|}{$B 2$} & \multicolumn{3}{|c|}{$B 3$} \\
\hline \multirow{3}{*}{ A } & $(0$ & 8 & $0)$ & $(0$ & 0 & 0 & $(0$ & 0 & 0 ) & 0 & 0 & 0 \\
\hline & & 1 & & 0 & 1 & & & 1 & & 0 & 0 & 1 \\
\hline & 0 & 128 & $0)$ & 0 & 0 & 0 & 0 & 0 & 0 & 0 & 0 & 0 \\
\hline \multirow{3}{*}{ B1 } & $(1$ & 1 & 1) & 0 & 8 & $0)$ & $(0$ & 1 & $1)$ & $(0$ & 0 & $1)$ \\
\hline & 1 & 1 & & & 1 & & 0 & 0 & & 0 & 0 & 0 \\
\hline & 1 & 1 & 1) & 0 & 128 & 0 & 0 & 0 & $0)$ & 0 & 0 & $0)$ \\
\hline \multirow{3}{*}{ B2 } & $(1$ & 1 & 1) & 0 & 0 & 0 & 0 & 8 & $0)$ & 0 & 0 & $1)$ \\
\hline & 1 & 1 & 1 & 0 & 0 & 0 & & 1 & & 0 & 0 & 1 \\
\hline & 1 & 1 & 1) & 1 & 1 & 0 & 0 & 128 & $0)$ & 0 & 0 & $0)$ \\
\hline \multirow{3}{*}{ B3 } & $(1$ & 0 & $0)$ & 0 & 0 & $0)$ & 0 & 0 & $0)$ & 0 & 8 & $0)$ \\
\hline & 1 & 0 & 0 & & 0 & & & 0 & & & 1 & 2 \\
\hline & 1 & 0 & 0 & 1 & 0 & 0 & 1 & 0 & & 0 & 128 & 0 \\
\hline
\end{tabular}

Spatial data retrieval algorithm based on sketch. The program of Spatial-Query-by-Sketch can be formally described as a standard binary constraint satisfaction problem:

( 1 ) A finite collection of $\mathrm{N}$ variables in the sketch, $\mathrm{V} 1, \mathrm{~V} 2, \mathrm{~V} 3, \ldots \mathrm{Vn}$;

( 2 ) For each variable $\mathrm{Vi}$, a finite domain of Vi values, $\mathrm{Di}=\left\{v_{1}^{i}, v_{2}^{i}, \ldots, v_{k i}^{i}\right\}$;

( 3 )For each pair of variables ( $\mathrm{Vi}, \mathrm{Vj}$ ), a constraint $\mathrm{C}\{\mathrm{i}, \mathrm{j}\}$ between $\mathrm{Di}$ and $\mathrm{Dj}$ which is simply a subset of $\operatorname{Dix} \mathrm{Dj}$. if $\left(V_{l}^{i}, V_{m}^{j}\right) \in \mathrm{C}\{\mathrm{i}, \mathrm{j}\}$ we say that the assignment $\left\{\mathrm{Vi} \leftarrow V_{l}^{i}, \mathrm{Vj} \leftarrow V_{m}^{j}\right\}$ is consistent.

The goal of Spatial-Query-by-Sketch is to find a solution such that for all $\mathrm{i}, \mathrm{j},\left\{\mathrm{Vi} \leftarrow v_{1}^{i}, \mathrm{Vj} \leftarrow v_{m}^{j}\right\}$ is consistent. To solve the constraint satisfaction problem, many algorithms have been proposed. Among them, one of the most effective algorithms is the forward checking algorithm(FC).

The forward checking algorithm constructs solutions by considering assignments to variables in a particular order, which for concreteness we take to be V1, V2, V3,...Vn.suppose that we have found a consistent assignment to the first i-1 variables, which means that all pairwise comparisons involving only these i-1 variables are satisfied. At this point, we call V1, V2, V3,...Vi-1 the past variables, Vi the current variable, and the others the future variables. The characteristic data structure of the FC algorithm is a two dimensional array Domain. The idea is that Domain ${ }_{j}^{i}$ will contain 0 if and only if the assignment $\mathrm{Vi} \leftarrow V_{j}^{i}$ is consistent with the assignments chosen for all the past variables. Otherwise, it contains the index of the first assigned variable with which $\mathrm{Vi} \leftarrow V_{j}^{i}$ is inconsistent.

It follows that,when we are considering a possible value $v_{l}^{i}$ for the current variable $\mathrm{Vi}$,it is sufficient to look for a zero in Domai $n_{1}^{i}$. Any such value is guaranteed to be consist with all past choices. When we make a successful assignment to the current variable, we must check it against all outsanding values of the future variables, updating Domain as necessary.

Analysis shows that the algorithm complexity of $\mathrm{FC}$ is $\mathrm{O}(\mathrm{KN})$ when the number of variables is $\mathrm{N}$, the largest for the domain is $K$, this is far away from the requirements of the practical application.During the process of query, the objects in the sketch are often adjacent objects in the map, according to this feature we can reduce the domain size of the variables, each time select the objects with the previous relationship with the neighboring fields as the next variable, which can significantly improve the efficiency.In order to apply the method to optimize the search algorithm the following definition of spatial neighborhood relations is given based on Voronoi diagram. 
Definition 1. $\mathrm{P}$ is a set of spatial objects $\mathrm{P}=\{\mathrm{P} 1, \mathrm{P} 2, \mathrm{P} 3, \ldots, \mathrm{Pn}\} \subseteq \operatorname{IR} 2 \quad(1 \leq \mathrm{n} \leq \infty)$, $\mathrm{Pi}, \mathrm{Pj} \in \mathrm{P}(\mathrm{i} \neq \mathrm{j}, \mathrm{i}, \mathrm{j}=1,2,3, \ldots \mathrm{n})$, If $\mathrm{Pi}$ meets $\mathrm{Pj}$ through at least $\mathrm{k}$ Voronoi regions then the distance between $\mathrm{Pi}$ and $\mathrm{Pj}$ is $\mathrm{k}$, Denoted as $\mathrm{d}(\mathrm{Pi}, \mathrm{Pj})=\mathrm{k}$, and the relationship between $\mathrm{Pi}$ and $\mathrm{Pj}$ is called $\mathrm{k}$-order neighborhood relations.

According to the definition, when querying spatial data by sketch, the current variable Vi instantiated with the values $V_{l}^{i}$, the next instance of the object does not need to consider all the domain, but only need to consider those instances which have 1-order neighborhood relations with the current assignment.

Experiment. A prototype of Spatial-Query-by-Sketch is under development with methods presented in this paper using C\#. The experimental database includes 1700 spatial scenes and is composed of two types of spatial scenes. the experimental results are in Table 3. We take four group of spatial scenes from the spatial database in first experiment. The precision and recall of the algorithm is up to $100 \%$ in the experiment, but when the number of samples which contain more objects, the efficiency decreased slightly. In this experiment the precision and recall has been able to reach $100 \%$ because the samples of this experiment are all taken from the spatial scene database and all objects are 1-order neighbors.

Table 3. the precision and recall of the first experiment

\begin{tabular}{ccccc}
\hline & $\begin{array}{c}\text { Samples } \\
\text { counts }\end{array}$ & $\begin{array}{c}\text { First } \\
\text { type }\end{array}$ & $\begin{array}{c}\text { Second } \\
\text { type }\end{array}$ & $\begin{array}{c}\text { precision and } \\
\text { recall }\end{array}$ \\
\hline First group & 10 & 10 & 0 & $100 \%$ \\
Second group & 20 & 20 & 0 & $100 \%$ \\
Third group & 40 & 20 & 20 & $100 \%$ \\
Fourth group & 60 & 30 & 30 & $100 \%$ \\
fourth group & 94.60 & 96.51 & 99.50 & 99.70 \\
\hline
\end{tabular}

\section{Conclusions}

This paper presents a sketch-based spatial data retrieval method, gives the representation of the sketch and query algorithms and its optimization for practical application, the method can be used to process intuitive spatial data query in complex spatial scene. However, for large mass spatial data the processing efficiency of the method is to be improved. Future research are: (1) study of fuzzy sketch query method combined with shape, topology and direction based on the qualitative representation of the shape and spatial similarity; (2) application of this method embedded into the GIS.

\section{References}

[1] Egenhofer, Spatial-Query-by-Sketch[C], Proceedings of the IEEE symposium on visual languages, IEEE Computer Society, Washington DC, USA,1996.

[2] Egenhofer M.J. Query processing in Spatial-Query-by -Sketch[J]. Journal of Visual Languages and Computing, 1997.

[3] BLASER A D. Sketching spatial queries [D]. Maine: University of Maine,2000.

[4] Ferri, F., P. Grifoni, and M. Rafanelli. Querying by sketch geographical databases and ambiguities. Copenhagen, Denmark: Springer Verlag, Heidelberg, D-69121, Germany. 2005.

[5] Ferri,F.,P.Grifoni and M. Rafanelli. The sketch recognition and query interpretation by GSQL, a geographical sketch query language. Proceedings of the Fifth International Conference on Computer and Information Technology, 2005,34 - 38. 
[6] Caduff,D.and Egenhofer, Geo-mobile query-by-sketch. International Journal of Web Engineering and Technology, 2007. 3(2): 157-175. 